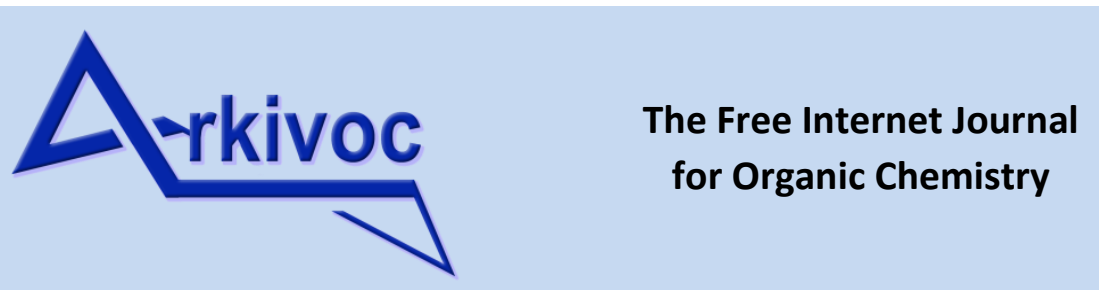

Archive for

Organic Chemistry
Paper

Arkivoc 2019, part vi, 0-0

to be inserted by editorial office

\title{
Synthesis, characterization and antibacterial studies of dichlorodiazadienes
}

Namig G. Shikhaliyev, ${ }^{* a}$ Gulnar T. Suleymanova, ${ }^{a}$ Aygun. A. İsrayilova, ${ }^{b}$ Khudaverdi. G. Ganbarov, ${ }^{b}$

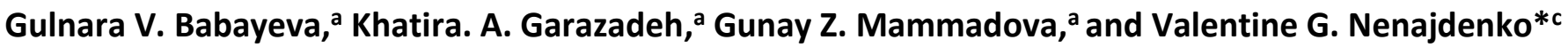

${ }^{a}$ Department of Organic Chemistry, Baku State University, Z. Khalilov str. 23, AZ 1148 Baku,Azerbaijan

${ }^{b}$ Department of Microbiology, Baku State University, Z. Khalilov str. 23, AZ 1148 Baku, Azerbaijan

${ }^{c}$ Department of Chemistry, Moscow State University, Leninskie Gory, Moscow, Russia

Email: namiqst@gmail.com, nenajdenko@gmail.com

\section{Abstract}

The synthesis of dichlorodiazadienes containing nitro and methyl groups was performed starting from phenylhydrazones. The role of functional groups in the crystal design was investigated by X-ray crystallographic analysis. Pnicogen $(\mathrm{N} . . . \mathrm{Cl})$, halogen $(\mathrm{Cl} . . . \mathrm{O})$ and halogen-halogen $(\mathrm{Cl} . . \mathrm{Cl})$ bonds were found in the synthesized compounds. Antibacterial studies were performed against five bacterial strains and potent antibacterial activity was detected against A.baumanii BDU32 and S.aureus BDU23.
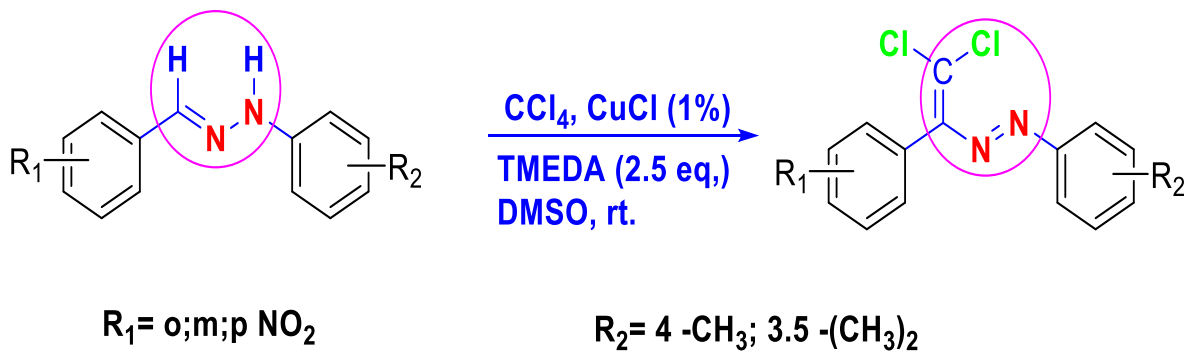

$\mathrm{R}_{2}=4-\mathrm{CH}_{3} ; 3.5-\left(\mathrm{CH}_{3}\right)_{2}$

Keywords: Dichlorodiazadienes, phenylhydrazones, catalytic olefinization, antibacterial activity, pnicogen and halogen non-covalent bonds 


\section{Introduction}

1,1-Dichlorodiazadienes are a valuable class of electrophiles. These compounds can be prepared using the reaction of phenylhydrazones of aldehydes in the presence of $\mathrm{CuCl}$ catalyst. ${ }^{1-6}$ Recently we demonstrated that these compoundsare interesting diazodyes. ${ }^{7,8}$ In addition, some compounds of this type exhibit antimicrobial properties. ${ }^{9,10}$

The nitro group is one of the most fundamental functional groups due to its strong electron-withdrawing properties. It is a key component of some drugs, dyes, explosive substances and other high-energy materials. ${ }^{11}$ Nitroaromatic compounds have been found in a number of natural products, including bacteria, fungi, and plants. ${ }^{11}$ This paper describes the synthesis of a family of dichlorodiazadienes containing a nitrophenyl component. The methodology used is based on the copper-catalyzed reaction of carbon tetrachloride with arylhydrazones derived from o-, $\mathrm{m}$-, and $\mathrm{p}$-nitrobenzaldehyde and 4-methylphenylhydrazine (Scheme 1). The target products were prepared in up to $80 \%$ isolated yields.

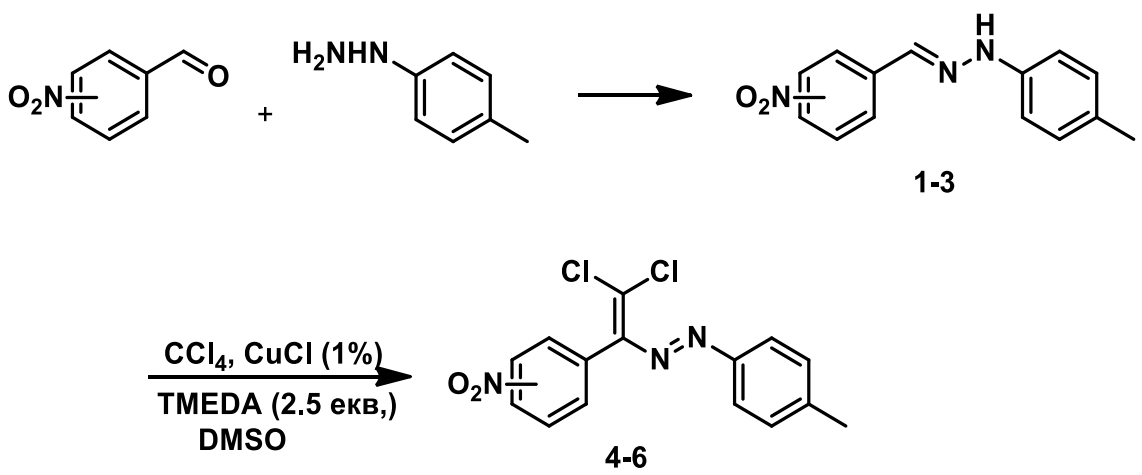

Scheme 1. The synthesis of (E)-1-(2,2-dichloro-1-(nitrophenyl))-2-(p-tolyl) diazene.

To develop a broader family of dichlorodiazadienes, we also replaced 4-methylphenylhydrazine with 3,5dimethylphenhydrazine, keeping the aldehydes the same (Scheme 2). As a result, a new set of diazadienes was synthesized in up to $65 \%$ yield.

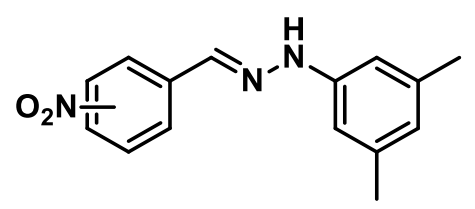

7-9

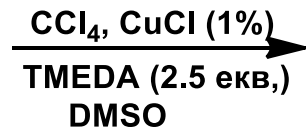

DMSO

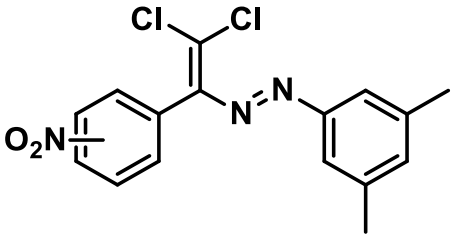

10-12

Scheme 2. The synthesis of (E)-1-(2,2-dichloro-1-(nitrophenyl) vinyl)-2-(3,5-dimethylphenyl) diazenes.

Compounds 1-12 were synthesized according to the reported method. ${ }^{1}$ The antibacterial activity of several synthesized compounds $(4,5,6,10,11$, and $\mathbf{1 2})$ was studied by agar well diffusion method. ${ }^{12}$ Morever, the minimum inhibitory concentration (MIC) of the compounds was determined by the two-fold microdilution method. ${ }^{13-15}$ 


\section{Results and Discussion}

Structures of the synthesized compounds have been studied by a number of analytical methods, one of which was X-ray crystallographic method. The molecular structures of compounds 4, 5, 6, 10 and 11 are presented in Figure 1. Crystallographic data for the structural analysis have been deposited in the Cambridge Crystallographic Data Center (CCDC 1841658 for 4, 1897671 for 5, 1897670 for 6, 1898374 for 10, and 1898098 for 11). Copies of this information can be obtained free of charge from The Director, CCDC, 12 Union Road, Cambridge CB2 1EZ, UK (Fax: (+44) 1223-336033; Email: deposit@ccdc.cam.ac.uk or www.ccdc.cam.ac.uk/data request/cif). The crystallographic-structure data of the substances are presented in Table 1.

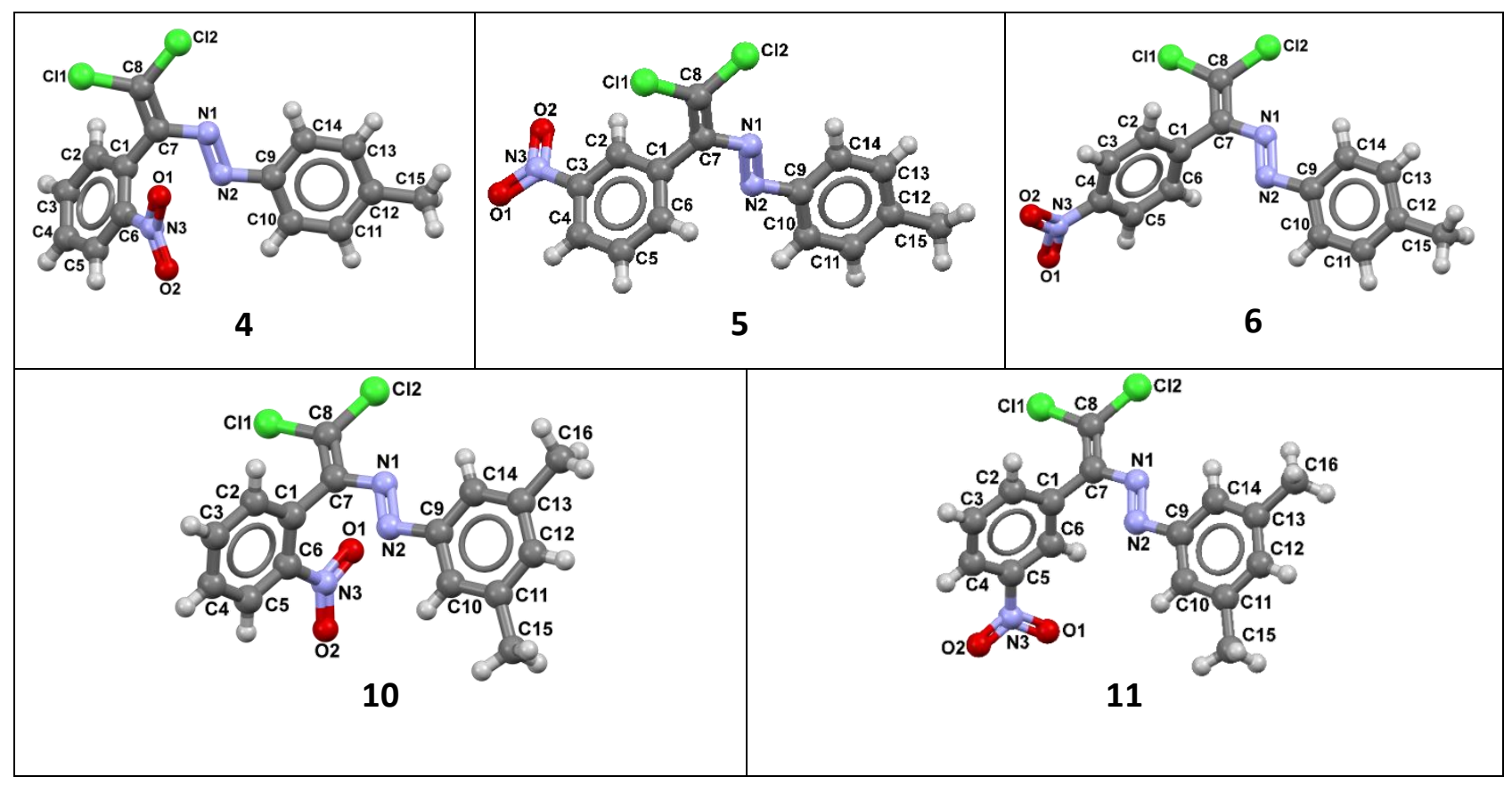

Figure 1. X-ray crystallographic structures of dichlorodiazadienes 4, 5, 6, 10 and 11. 
Table 1. X-ray crystallographic data for 4, 5, 6, 10 and 11

\begin{tabular}{|c|c|c|c|c|c|}
\hline & 4 & 5 & 6 & 10 & 11 \\
\hline $\begin{array}{l}\text { Empirical } \\
\text { formula }\end{array}$ & \multicolumn{2}{|c|}{$\mathrm{C}_{15} \mathrm{H}_{11} \mathrm{Cl}_{2} \mathrm{~N}_{3} \mathrm{O}_{2} \mathrm{C}_{15} \mathrm{H}_{11} \mathrm{Cl}_{2} \mathrm{~N}_{3} \mathrm{O}_{2}$} & \multicolumn{2}{|c|}{$\mathrm{C}_{15} \mathrm{H}_{11} \mathrm{Cl}_{2} \mathrm{~N}_{3} \mathrm{O}_{2} \mathrm{C}_{16} \mathrm{H}_{13} \mathrm{Cl}_{2} \mathrm{~N}_{3} \mathrm{O}_{2}$} & $\mathrm{C}_{16} \mathrm{H}_{13} \mathrm{Cl}_{2} \mathrm{~N}_{32}$ \\
\hline$F w$ & 336.17 & 336.17 & 336.17 & 350.19 & 350.19 \\
\hline Cryst. Syst. & Triclinic & Monoclinic & $\begin{array}{l}\text { Orthorhom } \\
\text { bic }\end{array}$ & Monoclinic & Triclinic \\
\hline Space group & P-1 & $\mathrm{P} 21 / \mathrm{C}$ & $\mathrm{Pna}_{1}$ & $\mathrm{P} 22_{1} / \mathrm{C}$ & P-1 \\
\hline$a(\AA)$ & $8.3927(14)$ & $7.971(3)$ & $13.904(2)$ & $14.8374(2)$ & $8.0857(6)$ \\
\hline$b(\AA)$ & $14.112(3)$ & $29.176(9)$ & $13.443(2)$ & $8.50440(10$ & $10.0229(7)$ \\
\hline$c(\AA)$ & $14.251(3)$ & $7.083(2)$ & $8.4016(17)$ & $13.37370(10)$ & $11.9447(9)$ \\
\hline$\alpha^{0}$ & $72.648(5)$ & 90 & 90 & 90 & $114.722(2)$ \\
\hline$\beta,{ }^{0}$ & $89.988(6)$ & $111.510(12)$ & 90 & $104.7220(10)$ & $94.057(3)$ \\
\hline$\gamma_{1}^{0}$ & $80.342(6)$ & 90 & 90 & 90 & $102.360(3)$ \\
\hline$V,\left(\AA^{3}\right)$ & $1586.0(5)$ & $1532.4(8)$ & $1570.3(5)$ & $1632.13(3)$ & $844.80(11)$ \\
\hline$\rho_{\text {calc }}\left(\mathrm{g} \mathrm{cm}^{-3}\right)$ & 1.408 & 1.457 & 1.422 & 1.425 & 1.377 \\
\hline $\begin{array}{ll}\mu(M o & K \alpha) \\
\left(\mathrm{mm}^{-1}\right) & \end{array}$ & 0.418 & 0.433 & 0.423 & 3.689 & 0.396 \\
\hline$Z$ & 4 & 4 & 4 & 4 & 2 \\
\hline$R 1^{a}(I \geq 2 \sigma)$ & 0.0720 & 0.0656 & 0.0325 & 0.0395 & 0.0439 \\
\hline$w R 2^{b}(I \geq 2 \sigma)$ & 0.1559 & 0.1638 & 0.0809 & 0.1095 & 0.1132 \\
\hline GOOF & 1.015 & 1.087 & 1.038 & 1.044 & 1.039 \\
\hline
\end{tabular}

${ }^{a} R 1=\Sigma|| F_{o}|-| F_{c}|| / \Sigma\left|F_{o}\right|$.

${ }^{b} W R 2=\left[\Sigma\left[w\left(F_{o}^{2}-F_{c}^{2}\right)^{2}\right] / \Sigma\left[w\left(F_{o}^{2}\right)^{2}\right]\right]^{1 / 2}$.

Non-covalent interactions were detected between molecules by X-ray diffraction. In recent years, extensive research has been carried out for studying the role of non-covalent bonds in the design and synthesis of new compounds. Different types of non-covalent bonds were observed depending on the position of the nitro-group as was demonstrated using compounds $\mathbf{1 0}$ and $\mathbf{1 1}$. In these examples, there are non-covalent intermolecular and intramolecular bonds in the the crystal structure (Figure 2). Intermolecular $\mathrm{Cl}$... Cl bonds $[3.485 \AA$ ] and intramolecular $\mathrm{Cl} \bullet \bullet \mathrm{N}$ pnicogen bonds $[2.930 \AA]$ were observed in compound 10 . These distances between atoms are shorter than the sum of the Van der Vaals radius $(\mathrm{Cl}+\mathrm{Cl}=1.75+1.75=3.5 \AA),(\mathrm{N}+\mathrm{Cl}=1.55$ $+1.75=3.30 \AA$ ) . At the same time, for compound 11 , along with intramolecular pnicogen $\mathrm{Cl}$...N bonds [2.926 $\AA$ ] , intermolecular halogen $\mathrm{Cl}$...O bonds [2.984, $3.301 \AA]$ were found, which were smaller than the sum of the Van der Vaals radius $(\mathrm{Cl}+\mathrm{O}=1.75+1.52=3.27 \AA$ ). Thus, both intermolecular and intramolecular non-covalent bonds play an important role in the formation of the crystalline structures of compounds 10 and 11. 


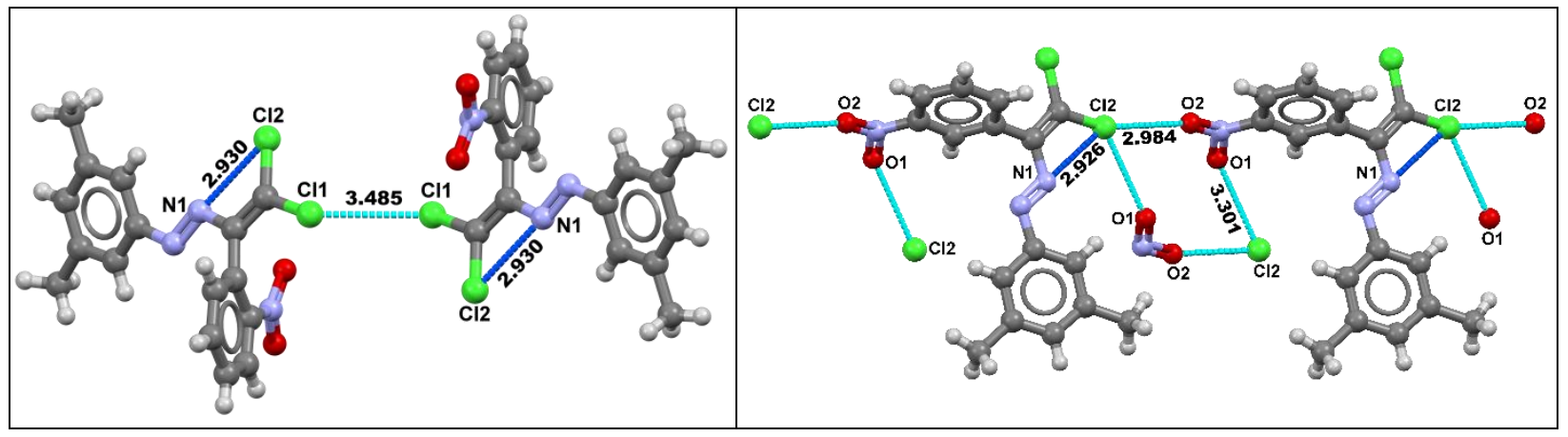

Figure 2. Non-covalent bonds are shown with fractured lines.

Next, antibacterial activity of the prepared compounds was screened against Escherichia coli BDU12, Acenitobacter baumanii BDU32, Pseudomonas aeruginosa BDU49, Klebsiella pneumonia BDU44 and Staphylococcus aureus BDU23. The compounds were evaluated at a concentration of $0.2 \%$ in DMSO by agar well diffusion method ${ }^{12}$. The zone of inhibition was measured and the results obtained are shown in Table 2. Compound $\mathbf{5}$ showed moderate activity against bacterial strains and better activity was detected only against P.aeruginosa BDU49. From the results, it was revealed that the antibacterial activity of compound $\mathbf{1 1}$ was better against the various bacterial strains than the other compounds. As shown in Table 2, minimal activity against A.baumanii BDU32 and S.aureus BDU23 bacterial strains was detected in the case of compound 5, whereas, compound $\mathbf{1 1}$ showed better activity when tested against A.baumanii BDU32 and S.aureus BDU23. Maximum and minimal antibacterial effects of compounds $\mathbf{4}$ and $\mathbf{1 0}$ were found against K.pneumoniae BDU 44 and S, aureus BDU23, respectively. The screening results revealed that activity of compound 6 was weaker than other tested compounds. Moreover, compound 6 was inactive against A.baumanii BDU32 and S.aureus BDU23, and its maximum activity was observed against K.pneumoniae BDU44. Compound $\mathbf{1 2}$ has better activity towards A.baumanii BDU32 and S.aureus BDU23 as compared with compound 6, whereas 6 is more active in the case of P.aeruginosa BDU49. In the case of P.aeruginosa BDU49, we can order the antibacterial activity of the compounds shown below as $11>12>5>4>10>6$.

Table 2. Antibacterial activity of compounds [diameter of inhibition zone $(\mathrm{mm})$ ] against bacterial strains

\begin{tabular}{cccccc}
\hline \multirow{2}{*}{ Compound } & $\begin{array}{c}\text { B.baumanii } \\
\text { BDU32 }\end{array}$ & $\begin{array}{c}\text { E.coli } \\
\text { BDU12 }\end{array}$ & $\begin{array}{c}\text { K.pneumoniae } \\
\text { BDU44 }\end{array}$ & $\begin{array}{c}\text { P.aeruginosa } \\
\text { BDU49 }\end{array}$ & $\begin{array}{c}\text { S.aureus } \\
\text { BDU23 }\end{array}$ \\
\cline { 2 - 6 } & $11.0 \pm 0.5$ & $15.5 \pm 0.6$ & $14.5 \pm 0.6$ & $17.0 \pm 0.8$ & $11.0 \pm 0.5$ \\
$\mathbf{5}$ & $19.5 \pm 0.7$ & $16.7 \pm 0.8$ & $17.6 \pm 0.8$ & $18.3 \pm 1.0$ & $20.3 \pm 0.7$ \\
$\mathbf{1 1}$ & $16.3 \pm 0.3$ & $14.3 \pm 0.7$ & $16.7 \pm 0.5$ & $15.7 \pm 0.7$ & $13.3 \pm 0.4$ \\
$\mathbf{4}$ & $16.0 \pm 0.5$ & $16.3 \pm 0.3$ & $16.7 \pm 0.4$ & $15.3 \pm 0.5$ & $13.7 \pm 0.5$ \\
$\mathbf{6}$ & - & $12.3 \pm 0.4$ & $15.3 \pm 0.6$ & $12.7 \pm 0.6$ & - \\
$\mathbf{1 2}$ & $15.3 \pm 0.7$ & $16.0 \pm 0.5$ & $16.3 \pm 0.7$ & $18.7 \pm 0.6$ & $14.7 \pm 0.6$ \\
\hline
\end{tabular}

After initial screening, the antibacterial activity of the compounds using the minimum inhibitory concentration (MIC) was tested by the two-fold microdilution method ${ }^{15-17}$. As shown in Table 3 , the bacterial strains were more susceptible to compound $\mathbf{1 1}$ than the other compounds. Compound $\mathbf{1 1}$ had the highest inhibitory effect against A.baumanii BDU32 and S. aureus BDU23 in (value of $31.25 \mu \mathrm{g} / \mathrm{mL}$ ). 
Table 3. Minimum inhibitory concentration of compounds $(\mu \mathrm{g} / \mathrm{mL})$

\begin{tabular}{cccccc}
\hline \multirow{2}{*}{ Compound } & $\begin{array}{c}\text { Bacterial strains } \\
\text { A.baumanii }\end{array}$ & $\begin{array}{c}\text { E.coli } \\
\text { BDU32 }\end{array}$ & $\begin{array}{c}\text { K.pneumoniae } \\
\text { BDU12 }\end{array}$ & $\begin{array}{c}\text { P.aeruginosa } \\
\text { BDU44 }\end{array}$ & $\begin{array}{c}\text { S.aureus } \\
\text { BDU23 }\end{array}$ \\
\hline $\mathbf{5}$ & 500 & 1000 & 2000 & 500 & 1000 \\
11 & 31.25 & 1000 & 1000 & 500 & 31.25 \\
$\mathbf{4}$ & 500 & 1000 & 2000 & 500 & 2000 \\
10 & 1000 & 1000 & 2000 & 500 & 2000 \\
$\mathbf{6}$ & - & 1000 & 1000 & 500 & - \\
12 & 500 & 2000 & 2000 & 500 & 2000 \\
\hline
\end{tabular}

Moreover, compound 11 presented a MIC of $500 \mu \mathrm{g} / \mathrm{mL}$ for P.aeruginosa BDU49, half the value obtained with E.coli BDU12 and K.penumoniae BDU44. No inhibition was detected for compound 6 against S.aureus BDU23 and A.baumanii BDU32. The MIC values of compound 6 ranged from $500-1000 \mu \mathrm{g} / \mathrm{mL}$ for P. aeruginosa BDU49, E.coli BDU12, and K.penumoniae BDU44, respectively. E. coli BDU12 was more susceptible against all of the compounds $(1000 \mu \mathrm{g} / \mathrm{mL})$ except compound $12(2000 \mu \mathrm{g} / \mathrm{mL})$. All compounds reached the similar inhibitory effect $(500 \mu \mathrm{g} / \mathrm{mL})$ against P.aeruginosa BDU49. The MIC values of compounds $\mathbf{4 , 1 0}$ and 12 were identical (2000 $\mu \mathrm{g} / \mathrm{mL}$ ) in the case of S.aureus BDU23, and the MIC of compound 5 was two times lower than this value (1000 $\mu \mathrm{g} / \mathrm{mL}$ ). All tested compounds presented MIC values in the range of $1000-2000 \mu \mathrm{g} / \mathrm{mL}$ for K.pneumoniae BDU44. The MIC value of compounds 4, 5 and 12 was $500 \mu \mathrm{g} / \mathrm{mL}$ when tested with A.baumanii BDU32, while the value was $1000 \mu \mathrm{g} / \mathrm{mL}$ in the case of compound 10 .

\section{Conclusions}

A family of nitro-substituted 1,1-dichlorodiazadienes was prepared using the copper-catalysed (CuCl) reaction of phenylhydrazones of aldehydes with $\mathrm{CCl}_{4}$. Crystal structures of diazadienes were studied using $\mathrm{X}$-ray crystallography which demonstrated intermolecular and intramolecular non-covalent interactions in the crystals. Some of the prepared 1,1-dichlorodiazadienes exhibited prononced antibacterial activity.

\section{Experimental Section}

General. The syntheses of compounds were carried out at the Chemistry Department of Baku State University (Azerbaijan). The X-ray analyses of compounds 4, 5, 6, 10 and 11 were carried out using the Bruker APEX II CCD (T $296 \mathrm{~K}, \lambda$ MoK $\alpha$ - radiation, graphite monochromator, $\phi$ - and $\omega$-scan) diffractometer. NMR ${ }^{1} \mathrm{H}$ and ${ }^{13} \mathrm{C}$ spectra were recorded using a Bruker Avance 300 (working frequency is $300 \mathrm{MHz}$ ) using $\mathrm{CDCl}_{3}$ and DMSO solvents, respectively. $\mathrm{SiMe}_{4}$ (TMS) was used as an internal standard. Elemental Analysis was performed on a Carlo Erba 1108 Analyzer. Thin-layer chromatography (TLC) was performed on silhouette plate UB-254 and acidified $\mathrm{KMnO} 4$ solution; UV lamp rays were used to make spots visible. Column chromatography was performed on silica gel of Merk firm (63-200). All compounds were crystallized from a methylene chloride and hexane (1:3) solvent system. 
General procedure for synthesis of phenylhydrazones. Schiff bases 1-3 and 7-9 were synthesized according to the reported method. ${ }^{1-4} \mathrm{~A}$ mixture of (2-nitrophenyl) hydrazine (10.2 mmol), $\mathrm{CH}_{3} \mathrm{COONa}(0.82 \mathrm{~g})$, ethanol (50 $\mathrm{mL}$ ) and the corresponding 4-substituted aldehyde $(10 \mathrm{mmol})$ was refluxed at $80^{\circ} \mathrm{C}$ with stirring for $2 \mathrm{~h}$. The reaction mixture was cooled to room temperature and water $(50 \mathrm{~mL})$ was added to give a precipitate of crude product, which was filtered off, washed with diluted ethanol (1:1 with water) and dried under vacuum of a rotary evaporator.

(E)-1-(2-nitrobenzylidene)-2-(p-tolyl)hydrazine (1). Red solid (90\%); mp $141{ }^{\circ} \mathrm{C} .{ }^{1} \mathrm{H}$ NMR (300 MHz, DMSO ): $\delta$ $10.82(\mathrm{~s}, 1 \mathrm{H},-\mathrm{NH}), 8.22(\mathrm{~s}, 1 \mathrm{H},-\mathrm{CH}), 8.17(\mathrm{~d}, J 9 \mathrm{~Hz}, 1 \mathrm{H}$, arom), 7.97 (d, J $6 \mathrm{~Hz}, 1 \mathrm{H}$, arom), $7.72(\mathrm{t}, J 9 \mathrm{~Hz}, 1 \mathrm{H}$, arom), 7.50 (t, J $9 \mathrm{~Hz}, 1 \mathrm{H}$, arom), 7.08 (d, J $9 \mathrm{~Hz}, 2 \mathrm{H}$, arom), 7.02 (d, J 9Hz, 2H, arom), 2.50 (s, 3H, $\left.-\mathrm{CH}_{3}\right) .{ }^{13} \mathrm{C} \mathrm{NMR}$ (75 MHz, DMSO- $\left.d_{6}\right): \delta 162.3,147.0,142.7,133.6,130.5,130.3,128.9,128.4,127.3,125.0,112.9$, 20.7. Anal. Calcd for $\mathrm{C}_{14} \mathrm{H}_{13} \mathrm{~N}_{3} \mathrm{O}_{2}$ (M 241.25); Calculated \%: C, 65.87: $\mathrm{H}, 5.13 ; \mathrm{N}, 16.46$. Found \%: C, 65.84; $\mathrm{H}, 5.15 ; \mathrm{N}, 16.44$. (E)-1-(3-nitrobenzylidene)-2-(p-tolyl)hydrazine (2). Yellow solid, (90\%), mp $148{ }^{\circ} \mathrm{C} .{ }^{1} \mathrm{H} \mathrm{NMR}(300 \mathrm{MHz}, \mathrm{DMSO})$ $\delta 10.59(\mathrm{~s}, 1 \mathrm{H}, \mathrm{NH}), 8.41(\mathrm{~s}, 1 \mathrm{H}, \mathrm{CH}), 8.09-8.05(\mathrm{~m}, 2 \mathrm{H}, \mathrm{arom}), 7.92(\mathrm{~s}, 1 \mathrm{H}$, arom), $7.64(\mathrm{t}, 1 \mathrm{H}, J 9.1 \mathrm{~Hz}$, arom), 7.08-7.00 (m, 4H, arom), 2.22 (s, 3H, $\left.\mathrm{CH}_{3}\right) .{ }^{13} \mathrm{C}$ NMR (75 MHz, DMSO) $\delta 148.8,142.9,138.5,133.5,131.8,130.5$, 130.1, 128.5, 122.2, 119.8, 112.7, 20.7. Anal. Calcd for $\mathrm{C}_{14} \mathrm{H}_{13} \mathrm{~N}_{3} \mathrm{O}_{2}$ (M 241.25); Calculated \%: C, 65.87; $\mathrm{H}, 5.13$; $\mathrm{N}, 16.46$. Found \%: C, 65.85; H, 5.17; N, 16.47 .

(E)-1-(4-nitrobenzylidene)-2-(p-tolyl)hydrazine (3). Orange solid (87\%), mp $152^{\circ} \mathrm{C} .{ }^{1} \mathrm{H} \mathrm{NMR} \mathrm{(300} \mathrm{MHz,} \mathrm{DMSO)} \delta$ $10.82(\mathrm{~s}, 1 \mathrm{H}, \mathrm{NH}), 8.21$ (d, 2H, J $6.2 \mathrm{~Hz}$, arom ), $7.88(\mathrm{~d}, 2 \mathrm{H}, \mathrm{J} 6.2 \mathrm{~Hz}$, arom), $7.84(\mathrm{~s}, 1 \mathrm{H}, \mathrm{CH}) 7.06$ (s, 4H, arom), $2.23\left(\mathrm{~s}, 3 \mathrm{H}, \mathrm{CH}_{3}\right) .{ }^{13} \mathrm{CNMR}(75 \mathrm{MHz}$, DMSO) $\delta 162.3,146.3,143.2,142.6,133.3,130.1,129.1,126.3,124.5,113.0$, 20.8. Anal. Calcd for $\mathrm{C}_{14} \mathrm{H}_{13} \mathrm{~N}_{3} \mathrm{O}_{2}$ (M 241.25); Calculated \%: C, 65.87; H, 5.13; N, 16.46. Found \%: C, 65.84; $\mathrm{H}$, $5.16 ; \mathrm{N}, 16.42$.

(E)-1-(3,5-dimethylphenyl)-2-(2-nitrobenzylidene)hydrazine (7). Purple solid (92\%), mp $147^{\circ} \mathrm{C} .{ }^{1} \mathrm{H}$ NMR (300 $\left.\mathrm{MHz}, \mathrm{DMSO}-d_{6}\right) \delta 10.74(\mathrm{~s}, 1 \mathrm{H}, \mathrm{NH}), 8.20(\mathrm{~s}, 1 \mathrm{H}, \mathrm{CH}-), 8.13(\mathrm{~d}, J 7.9 \mathrm{~Hz}, 1 \mathrm{H}$, arom), 7.93 (d, J $9.0 \mathrm{~Hz}, 1 \mathrm{H}$, arom), 7.68 (t, J $6.2 \mathrm{~Hz} 1 \mathrm{H}$, arom), 7.45 (t, J $7.6 \mathrm{~Hz}, 1 \mathrm{H}$, arom), 2.21 (s, 6H, $\left.-\mathrm{CH}_{3}\right) .{ }^{13} \mathrm{C} \mathrm{NMR}(75 \mathrm{MHz}, \mathrm{DMSO}) \delta 147.0$, $144.8,138.7,133.6,130.6,130.4,128.5,127.3,124.9,122.2,110.7$, 21.6. Anal. Calcd for $\mathrm{C}_{14} \mathrm{H}_{13} \mathrm{~N}_{3} \mathrm{O}_{2}(M$ 269.30); Calculated \%: C, 66.90: H, 5.61; N, 15.60. Found \%: C, 66.93; H, 5.63; N, 15.64.

(E)-1-(3,5-dimethylphenyl)-2-(3-nitrobenzylidene)hydrazine (8). Orange solid (87\%), mp $169^{\circ} \mathrm{C} .{ }^{1} \mathrm{H}$ NMR (300 $\left.\mathrm{MHz}, \mathrm{DMSO}-d_{6}\right) \delta 10.55(\mathrm{~s}, 1 \mathrm{H}, \mathrm{NH}), 8.39(\mathrm{~s}, 1 \mathrm{H}, \mathrm{CH}), 8.09(\mathrm{~d}, J 9.6 \mathrm{~Hz}, 2 \mathrm{H}$, arom), $7.93(\mathrm{~s}, 1 \mathrm{H}, \operatorname{arom}),, 7.65(\mathrm{t}, J$ $8.0 \mathrm{~Hz}, 1 \mathrm{H}$, arom), $6.73\left(\mathrm{~s}, 2 \mathrm{H}\right.$, arom), $6.44\left(\mathrm{~s}, 1 \mathrm{H}\right.$, arom), $2.23\left(\mathrm{~s}, 6 \mathrm{H},-\mathrm{CH}_{3}\right) .{ }^{13} \mathrm{C} \mathrm{NMR}(75 \mathrm{MHz}, \mathrm{DMSO}) \delta 162.3$, $148.8,145.1,138.6,138.4,133.8,131.8,130.6,122.3,121.8,120.0,110.6,21.7$. Anal. Calcd for $\mathrm{C}_{14} \mathrm{H}_{13} \mathrm{~N}_{3} \mathrm{O}_{2}(M$ 269.30); Calculated \%: C, 66.90: H, 5.61; N, 15.60. Found \%: C, 66.92; H, 5.60; N, 15.62.

(E)-1-(3,5-dimethylphenyl)-2-(4-nitrobenzylidene) hydrazine (9). Red solid (93\%) mp $150{ }^{\circ} \mathrm{C} .{ }^{1} \mathrm{H} \mathrm{NMR}(300 \mathrm{MHz}$, DMSO-d $)) 10.74(\mathrm{~s}, 1 \mathrm{H}), 8.18(\mathrm{~d}, J 8.5 \mathrm{~Hz}, 2 \mathrm{H}), 7.88(\mathrm{~s}, 1 \mathrm{H}), 7.83(\mathrm{~d}, J 8.5 \mathrm{~Hz}, 2 \mathrm{H}), 6.77(\mathrm{~s}, 2 \mathrm{H}), 6.46(\mathrm{~s}, 1 \mathrm{H}), 2.23$ (s, 6H). ${ }^{13} \mathrm{C}$ NMR (75 MHz, DMSO) $\delta 146.3,144.8,143.1,138.7,133.6,126.3,124.4,122.3,110.9,31.2,21.6$. Anal. Calcd for $\mathrm{C}_{14} \mathrm{H}_{13} \mathrm{~N}_{3} \mathrm{O}_{2}$ (M 269.30); Calculated \%: C, 66.90; $\mathrm{H}, 5.61 ; \mathrm{N}, 15.60$. Found \%: C, 66.97; $\mathrm{H}, 5.62 ; \mathrm{N}$, 15.58.

General procedure for synthesis of dichlorodiazadienes. Diazenes 4-6 and 10-12 were synthesized according to the reported method. ${ }^{1-4}$ A 20-mL screw neck vial was charged with DMSO (10 mL), compounds 1-3, and 7-9 (1 $1 \mathrm{mmol})$, respectively, tetramethylethylenediamine (TMEDA) $(295 \mathrm{mg}, 2.5 \mathrm{mmol}), \mathrm{CuCl}(2 \mathrm{mg}, 0.02 \mathrm{mmol})$ and $\mathrm{CCl}_{4}$ (20 mmol, 10 equiv). After 1-3 hours (until TLC analysis showed complete consumption of the corresponding Schiff base), the reaction mixture was poured into an $\sim 0.01 \mathrm{M}$ solution of $\mathrm{HCl}(100 \mathrm{~mL}, \sim \mathrm{pH} 2-3)$, and extracted with dichloromethane $(3 \times 20 \mathrm{~mL})$. The combined organic phase was washed with water $(3 \times 50 \mathrm{~mL})$, followed by brine $\left(30 \mathrm{~mL}\right.$ ), dried over anhydrous $\mathrm{Na}_{2} \mathrm{SO}_{4}$ and concentrated in vacuo by rotary evaporator. The residue was 
purified by column chromatography on silica gel using appropriate mixtures of hexane and dichloromethane $(3 / 1-1 / 1)$, and the corresponding diazenes were obtained. IR spectra of all of the diazadienes contained adsorption bands at $1350-1360 \mathrm{~cm}^{-1}$ and $1520-1590 \mathrm{~cm}^{-1}$.

Single crystals of the dichlorodiazadienes were prepared from a mixture of $n$-hexaneand $\mathrm{CH}_{2} \mathrm{Cl}_{2}$ solvents by slow evaporation.

(E)-1-(2,2-dichloro-1-(2-nitrophenyl) vinyl)-2-(p-tolyl)diazene (4). Orange solid (74\%); mp 92 ${ }^{\circ} \mathrm{C} .{ }^{1} \mathrm{H}$ NMR (300 $\mathrm{MHz}, \mathrm{CDCl}_{3}$ ): $\delta 8.22$ (d, J $6 \mathrm{~Hz}, 1 \mathrm{H}$, arom), $7.68-7.22$ (m, 7H, arom ), 2.38 (s, 3H, $\left.-\mathrm{CH}_{3}\right) .{ }^{13} \mathrm{C} \mathrm{NMR}\left(75 \mathrm{MHz}, \mathrm{CDCl}_{3}\right)$ : $\delta 150.6,150.5,148.2,142.9,134.4,133.8,132.3,130.2,129.8,128.5,124.5,123.5,21.7$. Anal. Calcd for $\mathrm{C}_{15} \mathrm{H}_{11} \mathrm{Cl}_{2} \mathrm{~N}_{3} \mathrm{O}_{2}$ (M 336.17). Calculated \%: C, 53.59; $\mathrm{H}, 3.30 ; \mathrm{Cl}, 21.09 ; \mathrm{N}, 12.50$. Found \%: $\mathrm{C}, 53.55 ; \mathrm{H}, 3.37 ; \mathrm{Cl}$, 21.04; N, 12.56.

(E)-1-(2,2-dichloro-1-(3-nitrophenyl)vinyl)-2-(p-tolyl)diazene (5). Red solid (69\%), mp $141 \mathrm{C}^{0} .{ }^{1} \mathrm{H}$ NMR (300 $\left.\mathrm{MHz} \mathrm{CDCl}_{3}\right) \delta 8.30(\mathrm{~d}, 1 \mathrm{H}, J 9.1 \mathrm{~Hz}$, arom), $8.11(\mathrm{~d}, 1 \mathrm{H}, J 9.1 \mathrm{~Hz}$, arom), $7.68(\mathrm{~d}, 2 \mathrm{H}, J 6.1 \mathrm{~Hz}, \operatorname{arom}), 7.62(\mathrm{~d}, 1 \mathrm{H}, J$ $6.1 \mathrm{~Hz}$, arom), 7.53 (d, $1 \mathrm{H} . J 6.1 \mathrm{~Hz}$, arom), 7.27 (d, $2 \mathrm{H}, J 9.1 \mathrm{~Hz}, \operatorname{arom}), 2.42\left(\mathrm{~s}, 3 \mathrm{H}, \mathrm{CH}_{3}\right) .{ }^{13} \mathrm{C} \mathrm{NMR}\left(75 \mathrm{MHz} \mathrm{CDCl}_{3}\right)$ $\delta 161.7,150.7,150.2,148.1,143.0,136.4,134.4,129.9,129.2,125.4,123.7,123.4,21.6$. Anal Calcd for $\mathrm{C}_{15} \mathrm{H}_{11} \mathrm{Cl}_{2} \mathrm{~N}_{3} \mathrm{O}_{2}$ (M 336.17); Calculated \%: C, 53.59; $\mathrm{H}, 3.30 ; \mathrm{Cl}, 21.09 ; \mathrm{N}, 12.50$. Found \%: $\mathrm{C}, 53.52 ; \mathrm{H}, 3.35 ; \mathrm{Cl}$, 21.00; N, 12.54 .

(E)-1-(2,2-dichloro-1-(4-nitrophenyl)vinyl)-2-(p-tolyl)diazene (6). Red solid (80\%), mp $140^{\circ} \mathrm{C} .{ }^{1} \mathrm{H} \mathrm{NMR}(300 \mathrm{MHz}$, $\left.\mathrm{CDCl}_{3}\right) \delta 8.28(\mathrm{~d}, 2 \mathrm{H}, J 9.1 \mathrm{~Hz}$, arom), $7.70(\mathrm{~d}, 2 \mathrm{H}, J 9.1 \mathrm{~Hz}$, arom), 7.37 (d, 2H, J $9.1 \mathrm{~Hz}$, arom), 7.26 (d, $2 \mathrm{H}, J 9.1$ $\mathrm{Hz}$, arom), $2.42\left(\mathrm{~s}, 3 \mathrm{H}, \mathrm{CH}_{3}\right) .{ }^{13} \mathrm{C} \mathrm{NMR}\left(75 \mathrm{MHz}_{\mathrm{CDCl}}\right) \delta 150.8,150.5,147.9,143.1,139.7,135.4,131.3,129.9$, 123.4, 123.3, 21.6. Anal Calcd for $\mathrm{C}_{15} \mathrm{H}_{11} \mathrm{Cl}_{2} \mathrm{~N}_{3} \mathrm{O}_{2}$ (M 336.17); Calculated \%: C, 53.59; $\mathrm{H}, 3.30 ; \mathrm{Cl}, 21.09 ; \mathrm{N}, 12.50$. Found \%: C, 53.57; $\mathrm{H}, 3.31 ; \mathrm{Cl}, 21.06 ; \mathrm{N}, 12.48$.

(E)-1-(2,2-dichloro-1-(2-nitrophenyl)vinyl)-2-(3,5-dimethylphenyl) diazene (10). Orange solid, (60\%), mp 186 ${ }^{0} \mathrm{C} .{ }^{1} \mathrm{H}$ NMR $\left(300 \mathrm{MHz}, \mathrm{CDCl}_{3}-d\right) \delta 8.23$ (dd, J 8.0, $1.5 \mathrm{~Hz}, 1 \mathrm{H}$, arom), 7.67 (td, J 8.0, $1.4 \mathrm{~Hz}, 2 \mathrm{H}$, arom), 7.34 (dd, J 5.7, $1.6 \mathrm{~Hz}, 3 \mathrm{H}$, arom), 7.09 (s, $1 \mathrm{H}$, arom), 2.35 (s, 6H, CH 3$).{ }^{13} \mathrm{C} \mathrm{NMR}\left(75 \mathrm{MHz}, \mathrm{CDCl}_{3}\right) \delta$ 152.6, 150.4, 148.1, 138.7, 134.6, 133.7, 132.2, 130.0, 128.7, 124.5, 121.2, 31.6, 22.7, 21.1, 14.1. Anal Calcd for $\mathrm{C}_{15} \mathrm{H}_{11} \mathrm{Cl}_{2} \mathrm{~N}_{3} \mathrm{O}_{2}(M 350.20)$. Calculated \%: C, 54.87; H, 3.74; Cl, 20.25; N, 12.00. Found \%: C, 54.81; H, 3.75; Cl, 20.28; N, 12.04.

(E)-1-(2,2-dichloro-1-(3-nitrophenyl) vinyl)-2-(3,5-dimethylphenyl) diazene (11). Red solid (64\%), mp $165{ }^{\circ} \mathrm{C}$. ${ }^{1} \mathrm{H}$ NMR $\left(300 \mathrm{MHz} \mathrm{CDCl}_{3}-d\right) \delta 8.30(\mathrm{~d}, J 7.6 \mathrm{~Hz}, 1 \mathrm{H}$, arom), 8.10 (s, 1H, arom), 7.64 (t, J $7.6 \mathrm{~Hz}, 1 \mathrm{H}$, arom), 7.52 (d, J 7.6 Hz, 1H, arom), 7.39 (s, $2 \mathrm{H}$, arom), $7.13\left(\mathrm{~s}, 1 \mathrm{H}\right.$, arom), $2.38\left(\mathrm{~s}, 6 \mathrm{H}, \mathrm{CH}_{3}\right) .{ }^{13} \mathrm{C} \mathrm{NMR}\left(75 \mathrm{MHz}, \mathrm{CDCl}_{3}\right) \delta 162.3$, 152.7, 150.3, 148.1, 138.9, 136.4, 134.4, 133.9, 129.2, 125.4, 123.7, 121.15, 21.18. Anal Calcd for $\mathrm{C}_{15} \mathrm{H}_{11} \mathrm{Cl}_{2} \mathrm{~N}_{3} \mathrm{O}_{2}$ (M 350.20), Calculated \%: C, 54.87; H, 3.74; Cl, 20.25; N, 12.00. Found \%: C, 54.85; H, 3.76; Cl, 20.20; N, 12.03. (E)-1-(2,2-dichloro-1-(4-nitrophenyl) vinyl)-2-(3,5-dimethylphenyl) diazene (12). Red solid, (65\%), $\mathrm{mp} 138^{\circ} \mathrm{C}$. ${ }^{1} \mathrm{H} \mathrm{NMR}\left(300 \mathrm{MHz}, \mathrm{CDCl}_{3}-d\right) \delta 8.30$ (d, J $8.8 \mathrm{~Hz}, 2 \mathrm{H}$, arom), 7.37 (dd, J 6.8, 1.9 Hz, 4H, arom), 7.13 (s, 1H, arom), $2.38\left(\mathrm{~s}, 6 \mathrm{H}, \mathrm{CH}_{3}\right) .{ }^{13} \mathrm{C} \mathrm{NMR}\left(75 \mathrm{MHz}, \mathrm{CDCl}_{3}\right) \delta 152.8,150.6,147.9,139.8,138.9,133.9,131.3,123.4,121.1,100.0$, 21.2. Anal Calcd for $\mathrm{C}_{15} \mathrm{H}_{11} \mathrm{Cl}_{2} \mathrm{~N}_{3} \mathrm{O}_{2}$ (M 350.20). Calculated \%: $\mathrm{C}, 54.82 ; \mathrm{H}, 3.74 ; \mathrm{Cl}, 20.25 ; \mathrm{N}, 12.00$. Found \% $\mathrm{C}$, 54.89; $\mathrm{H}, 3.72 ; \mathrm{Cl}, 20.27 ; \mathrm{N}, 12.09$.

Antibacterial activity. Initial in vitro antibacterial activity of compounds 4, 5, 6, 10, 11, and 12 was screened against gram-positive Staphylococcus aureus BDU23 and gram-negative Escherichia coli BDU12, Acinetobacter baumanii BDU32, Pseudomonas aeruginosa BDU49, Klebsiella pneumonia BDU44 by the agar well diffusion method as described elsewhere. ${ }^{5}$ Bacterial strains were taken from culture collections of the Department of Microbiology of Baku State University (Azerbaijan). Firstly, the bacterial strains were grown in Muller Hinton broth overnight at $37{ }^{\circ} \mathrm{C}$. The optical density of the overnight bacterial cultures was adjusted to $0.5 \mathrm{McFarland}$ $\left(1.5 \times 10^{8} \mathrm{cfu} / \mathrm{mL}\right)$ by diluting in saline solution $(0.8 \% \mathrm{NaCl})$. Then $0.1 \mathrm{~mL}$ of this bacterial culture suspension was 
spread on sterile Muller Hinton Agar (MHA) plates and the plates were kept about $10 \mathrm{~min}$. After adsorption, the wells of $8 \mathrm{~mm}$ diameter were punched with the sterile tips or glass cork borer. The solution of test compounds $(0.2 \%)$, which were prepared freshly by dissolving of the chemicals in DMSO, were incorporated into the wells, and the plates were incubated at $37^{\circ} \mathrm{C}$ for $24 \mathrm{~h}$. After incubation, the antibacterial properties of test compounds were evaluated according to the diameter of the inhibition zone $(\mathrm{mm})$. Control experiments were carried out using DMSO solvent and no activity was detected against the studied bacterial strains. ${ }^{5}$

Determination of minimum inhibitory concentration (MIC). Minimum inhibitory concentrations of six compounds were determined based on CLSI by the two-fold microdilution method using 96-well microtiter plates (U-bottom). ${ }^{15-17}$ The tested compounds were prepared according to CLSI guidelines and diluted in microplates which contained Muller Hinton broth. At the end of microdilution, the compounds' concentrations ranged from 2000 to $15.625 \mu \mathrm{g} / \mathrm{mL}$. The inoculum was prepared freshly on Muller Hinton broth (MHB) ("Liofilchem") and bacterial strains [ $10^{5}$ colony forming units (CFU)] were inoculated to each well of the microplate and kept for incubation at $37{ }^{\circ} \mathrm{C}$ for $24 \mathrm{~h}$. After incubation, the growth of the bacterial strains was determined by the resazurin method. So, $30 \mu \mathrm{l}$ of resazurin solution (0.01\%) ("Sigma Aldrich") were added to each well and the microplates were left for incubation at $37{ }^{\circ} \mathrm{C}$ for about $4 \mathrm{~h}$. MIC is the lowest concentration of the tested compounds that inhibits the growth of the bacteria. In our assay, MIC was determined as the lowest concentration of the compounds which prevented the change in color from blue to pink since the color change from blue to pink indicated the growth of bacteria. ${ }^{18}$

\section{Acknowledgements}

This work was supported by the Science Development Foundation under the President of the Republic of Azerbaijan - Grant No EIF -BGM-4-RFTF-1/2017-21/13/4 and RFBR Grant N 18-53-06006.

\section{References}

1. Nenajdenko, V.G.; Shastin, A.V.; Gorbachev, V.M.; Shorunov, S. V.; Muzalevskiy, V.M.; Lukianova, A.I.; Dorovatovskii, P.V. ACS Catalysis, 2017, 7, 205-209.

https://doi.org/10.1021/acscatal.6b03196

2. Maharramov, A.M.; Suleymanova G.T.; Babayeva, G.V.; Ibragimova, Sh.A., Niyazova, A.A.; Shikhaliev, N.G.; Musayev, F.N.; Nenajdenko V.G. Chemical Problems Journal, 2018, 2, 230-238.

https://doi.org/10.32737/2221-8688-2018-2-230-238

3. Shastin, A.V.; Tsyrenova, B.D.; Sergeev, P.G.; Roznyatovsky, V.A.; Smolyar, I.V.; Khrustalev, V.N.; Nenajdenko V.G. Org. Lett., 2018, 20 (24), 7803-7806.

https://doi.org/10.1021/acs.orglett.8b03227

4. Shastin, A.V.; Sergeev, P.G.; Lukianova, A.I.; Muzalevskiy, V.M.; Khrustalev, V.N.; Dorovatovskii, P.V.; Nenajdenko V.G. Eur. J. Org. Chem., 2018, 36, 4996-5006

https://doi.org/10.1002/ejoc.201800441

5. Maharramov, A.M.; Suleymanova, G.T.; Babayeva, G.V.; Niyazova, A.A.; Mamedov, I.G.; Hasanova, U.A.; Shikhaliev, N.G.; Nenajdenko V.G. Journal of Low Dimensional Systems, 2018, 2 (1), 37-44.

6. Shikhaliyev, N. G.; Suleymanova, G.T.; Bagirova, Kh.N.; Asgerova, U.F.; Garazadeh, Kh.A.; Babayeva, G.V.; Ahmedova, N.E.; Nenajdenko, V.G. Journal of Low Dimensional Systems, 2018, 2 (2), 24-29. 
7. Shikhaliyev, N. Q. Ahmadova, N.E.; Gurbanov, A.V.; Maharramov, A.M.; Mammadova, G.Z.; Nenajdenko, V.G.; Zubkov, F.I.; Mahmudov, K.T.; Pombeiro A.J.L. Dyes and Pigments, 2018, 150, 377-381. https://doi.org/10.1016/i.dyepig.2017.12.033

8. Maharramov, A.M.; Shikhaliyev, N.Q.; Suleymanova, G.T.; Gurbanov, A.V.; Babayeva, G.V.; Mammadova, G.Z.; Zubkov, F.I.; Nenajdenko, V.G.; Mahmudov, K.T.; Pombeiro, A.J.L. Dyes and Pigments, 2018, 159, 135141.

https://doi.org/10.1016/j.dyepig.2018.06.022

9. Israyilova, A.; Mukhtarova, S.; Asgerova, U.; Shikhaliyev, N.; Ganbarov, Kh.; Maharramov, A. IJIRSET, 2017, 6, 21191-21195.

10. Maharramov, A.M.; Askerova, U.F.; Akhmedova, N.E.; Mukhtarova, S.Kh.; Garazadeh, Kh.A.; Shikhaliyev, N.G. Journal of Low Dimensional Systems, 2017, 1, 1-4.

11. Winkler, R.; Hertweck, C. Chembiochem, 2007, 8, 973-977. https://doi.org/10.1002/cbic.200700042

12. Hoshino, K.; Sato, K.; Akahane, K.; Yoshida, A.; Hayakawa, I.; Sato, M.; Une, T.; Osada, Y. Antimicrob. Agents Chemother. 1991, 35(2), 309-12.

https://doi.org/10.1128/AAC.35.2.309

13. Grabarczyk, M.; Mączka, W.; Żarowska B.; Maciejewska G.; Anioł, M; Journal of Saudi Chemical Society, 2018, 22 (3), 363-371.

https://doi.org/10.1016/j.jscs.2016.04.004

14. Tirkey, V.; Mishra, S.; Hirak, R.D.; Das, S.; Nayak, B.P.; Mobin, S.M.; Chatterjee, S. Journal of Organometallic Chemistry, 2013, 732, 122-129.

https://doi.org/10.1016/i.jorganchem.2013.02.020

15. CLSI-Clinical Laboratory Standards Institute. Methods for dilution antimicrobial susceptibility test for bacteria that grow aerobically, $11^{\text {th }}$ edition, 2018.

16. Martin, A.; Takiff, H.; Vandamme, P.; Swings, J.; Palomino, J.C.; Portaels, F. J. Antimicrob Chemother., 2006, 58, 327-331.

https://doi.org/10.1093/jac/dkl231

17. Israyilova, A.; Buroni S.; Forneris, F.; Scoffone, V. C.; Shixaliyev, N.Q.; Riccardi, G.; Chiarelli, L.R. PLos One, 2016, 11, 1-17.

https://doi.org/10.1371/journal.pone.0167350 